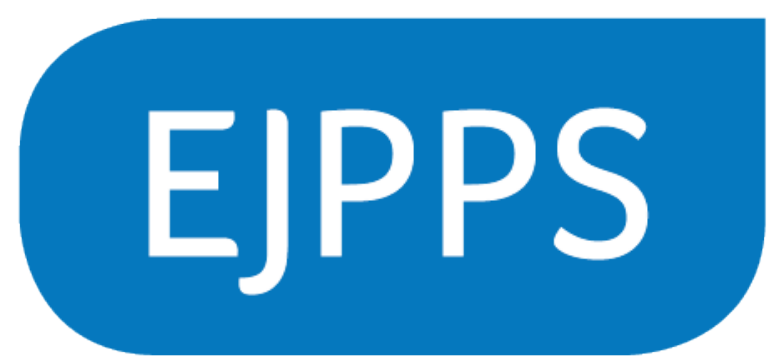

EUROPEAN JOURNAL OF
PARENTERAL AND
PHARMACEUTICAL SCIENCES

EJPPS - European Journal of Parenteral and Pharmaceutical Sciences Volume 26 Issue 2

https://www.ejpps.online/post/vol26-2-comparative-evaluation-of-amorphous-polymers-

in-solubility-and-bioavailability-enhancement

https://doi.org/10.37521/ejpps.26201

Comparative Evaluation of Amorphous Polymers in Solubility and Bioavailability Enhancement

Ankit Mishra, Priyanka Chaturvedi (Sagar Institute of Research and Technology ),

Pranali Mishra, MS Sudheesh (Department of Pharmaceutics, VNS Group of Institutions)

Corresponding Author: Dr Ankit Mishra

ORCID - 0000-0003-0795-8417

Associate Professor

Department of Pharmaceutics,

Faculty of Pharmacy,

VNS Group of Institutions,

Neelbud, Bhopal, MP, India

Pin-462044

Email: mishraaa@gmail.com

Telephone: +919827930533 


\title{
Comparative Evaluation of Amorphous Polymers in Solubility and Bioavailability Enhancement
}

\author{
Ankit Mishra, Priyanka Chaturvedi (Sagar Institute of Research and Technology), \\ Pranali Mishra, MS Sudheesh (Department of Pharmaceutics, VNS Group of Institutions)
}

\begin{abstract}
The present study aimed to enhance the dissolution rate, therefore bioavailability, of famotidine (FMT) using its solid dispersions (SDs) with polyvinyl pyrrolidone (PVP)-K 30, milk powder, and inulin, both in-vitro and in-vivo. The study was also aimed to compare the effect of different amorphous polymers in enhancing the dissolution rate of FMT. The SDs were prepared with a 1:4 weight ratio by a solvent evaporation technique. Evaluation of the properties of the SDs was performed using dissolution, Fourier-transform infrared (FTIR) spectroscopy, and X-ray diffraction (XRD) studies. The SDs of FMT exhibited an enhanced dissolution rate. The FTIR spectroscopic studies showed the stability of FMT and the absence of well-defined drug excipient interaction. The XRD studies indicated the amorphous state of FMT in SDs. The drug release rate of all SDs formulation was found to be greater than the pure drug. Within one hour of dissolution studies, $99.43 \%, 92.5 \%$, and $58.93 \%$ drug release were obtained, respectively, for PVP K-30, milk powder, and inulin. The first two were showing significantly higher release. SDs were also studied for bioavailability studies in-vivo in rats, which confirms that the SDs prepared by PVP K-30 and milk powder significantly enhancing the bioavailability of FMT. The maximum concentration of $15.05 \pm 2.45 \mu \mathrm{g} / \mathrm{ml}$ was achieved in 2 hours, and the area under the curve was found to be $33.78 \pm 7.3 \mu \mathrm{g}$. hour $/ \mathrm{ml}$. Therefore, the study results conclude that SDs of the FMT prepared by PVP K-30 successfully increases the dissolution and in-vivo bioavailability.
\end{abstract}

Keywords - Solid dispersion, Second generation solid dispersions, Famotidine, In-vivo bioavailability, amorphous polymers, dissolution enhancement, solubility enhancement.

\section{Introduction}

Famotidine (FMT) is a potent histamine $\mathrm{H} 2$ receptor antagonist used to treat peptic ulceration, reflux esophagitis, Zollinger-Ellison syndrome, and other conditions in which gastric acid reduction is beneficial. Additionally, it is less likely to bind to cytochrome P-450 or gastric alcohol dehydrogenase, so it has a low tendency for significant drug interactions. The preparation of solid dispersions (SD) is considered an excellent technique for increasing the bioavailability of poorly water-soluble drugs and increasing the dissolution rate. ${ }^{1}$ According to BCS (Biopharmaceutics Classification System), FMT is classified as a Class IV substance due to its poor solubility and permeability ${ }^{2}$. These characteristics can lead to reduced absorption and be a problem in achieving the therapeutic potential of these drugs, especially in oral administration. Several techniques have been developed to improve the bioavailability of such drugs. If not dissolved uniformly throughout the gastrointestinal tract (GIT), the drug leads to incomplete and variable absorption. Hence, a dosage form that achieves good solubility would be presented at the absorption site for improving its bioavailability and reducing its wastage ${ }^{3}$. 
Carrier selection is critical to the properties of the final solid dispersion. The solid dispersion was classified into crystalline solid dispersion and amorphous solid dispersion according to the physical state of the vehicles. Furthermore, the dispersions are also divided into four generations according to their composition. The first generation is the crystalline solid dispersion, which leads to a low dissolution rate due to the crystalline vector and low stability ${ }^{4}$. The second generation is an amorphous solid dispersion containing an amorphous solid solution (glass solutions) and amorphous solid suspensions based on the physical state of the drugs ${ }^{5}$. The third is the amorphous solid dispersion with surfactant additives or self-emulsifying agents capable of overcoming precipitation and recrystallization ${ }^{6}$.

The third solid dispersion contributes to the improvement of the dissolution rate and the decrease of supersaturation precipitation. The latest generation solid dispersion is a controlled release solid dispersion, and these transporters are insoluble or poorly dissolved in water so that they can support the release of water-soluble poor drugs ${ }^{7}$.

In the current work, PVP-K 30, milk powder, and inulin-based FMT SDs were prepared, using the solvent evaporation method with their respective carriers in various ratios and grinding time ${ }^{8}$. FMT has poor water solubility, which provides challenges in the formulation development process. The main reason for the inadequate bioavailability of the drug is its low dissolution rate and low solubility in an aqueous medium ${ }^{9}$. Today a large number of hydrophilic vehicles are discovered, which have shown significant results for improving solubility. Nowadays, most of the drug substances have been innovated, but the enterprise to improve the solubility and dissolution of hydrophobic drug substances remains one of the most challenging tasks in drug development. Dissolving the drug in an aqueous medium such as gastric fluid is essential to achieve better absorption and bioavailability of the orally administered drug. This study aims to compare the solid dispersion of FMT prepared from different polymers and amorphous agents. This study is aimed at estimating the pharmacokinetic parameters of the solid dispersion prepared from different polymers ${ }^{10}$.

\section{Materials and Methods}

\subsection{Material}

The gift sample of FMT was received from Ankur pharma (Baddi, India). PVP K-30 from Fishers, (India). Inulin from Acros organics (Mumbai, India). Milk powder was purchased from Nestle (India). Heparin was obtained from Biological E Ltd (Hyderabad, India). HPLC grade ethanol or HPLC grade acetonitrile was obtained from Loba Chemie (Mumbai, India) Hydrochloric acid, Himedia Pvt. Ltd (India) and all the other chemicals and solvents used were of pharmaceutical and analytical grade.

\subsection{Preparation of solid dispersion}

SDs containing FMT with PVP K-30 (fishers), milk powder, and inulin were prepared by the solvent evaporation method and respectively denoted by F1, F2, and F3 throughout the study. The ratio of drug and polymer was fixed at 1:4. The drug and carrier were mixed in ethanol and kept in a magnetic stirrer until the complete removal of solvent. The solid mass was lyophilized for complete drying and removal of the solvent. The solid mass was pulverized and passed through a sieve of 250 $\mu \mathrm{m}$ to get uniformly sized particles ${ }^{11},{ }^{12}$. The poly-dispersibility index confirmed uniformity in size via Horiba SZ-100 particle size analyzer; the value of $<0.6$ was considered optimum.

\subsection{Determination of percent drug content:}

The SDs equivalents to $10 \mathrm{mg}$ of FMT were dissolved separately in $100 \mathrm{ml}$ of $0.1 \mathrm{~N} \mathrm{HCl}$. The solution was filtered and further diluted so that the absorbance fell within the range of the standard curve. The samples were filtered through a $0.45 \mu \mathrm{m}$ membrane filter, and the drug content was 
determined spectro-photometrically at $266.5 \mathrm{~nm}$. UV spectroscopy study of other excipients was performed in the expected concentration of $5-50 \mu \mathrm{g} / \mathrm{ml}$ for the confirmation of interference in the UV range, and no significant absorbance was found in the range of absorbance of the drug.

\subsection{Percent practical yield:}

Percentage practical yield was calculated to know the efficiency of any method, thus its help in the selection of the appropriate method of production. SDs were collected and weighed to determine practical yield (PY) from the following equation-

PY $(\%)=[$ Practical Mass (Solid dispersion) $/$ Theoretical Mass (Drug+ Carrier) $\times 100$

\subsection{Saturation solubility studies}

Equivalent amounts of SD formulations were placed in a flask with a glass stopper containing $0.1 \mathrm{~N}$ $\mathrm{HCl}$, distilled water, and ethanol. The samples were placed on a shaker incubator, agitated at $37 \pm$ 0.5 ㅇ․ The aliquots were removed at $0,30,60$, and 180 mins until saturation was achieved. Aliquots were filtered through Whatman filter paper. The filtered samples were diluted and assayed using a UV visible spectrophotometer against a blank prepared ${ }^{13},{ }^{14}$.

\subsection{FT-IR Spectroscopy}

FT-IR spectroscopies of compounds were performed for studying the interaction between materials. FTIR is also helpful for the identification of the compound. FT-IR Spectroscopy of FMT, polymers, physical mixture, and the formulation was done using diamond crystals. Various peaks in the FT-IR spectrum were interpreted to identify different groups in the structure of the drug and their mixture. FT-IR spectroscopy can also be used to investigate and predict any physicochemical interactions between different components. ${ }^{15}$

\subsection{Powder X-ray diffraction (XRD)}

All powder samples were examined by wide-angle XRD using a copper target and nickel filter. Powder XRD of drug, polymers, and formulations was done with the voltage of $40 \mathrm{kV}$ and the current of $40 \mathrm{~mA}$; samples were analyzed ranging at room temperature over a range of 2-60 with a sampling interval of 0.0102 and a dwell time of 2 seconds $^{16}$.

\subsection{In-vitro dissolution study}

A dissolution apparatus II of the USP (paddle) type was used in this study. An appropriate amount of solid dispersion with particle size 120 to 150 mesh, physical mixtures containing 10 mg of FMT, was taken in the dialysis bag MW 1000-5000 Dalton. The SDs were placed into the apparatus before the initiation of the dissolution testing. Dissolution was performed at $37 \pm 0.5 \circ \mathrm{C}$ and $50 \mathrm{rpm}$. To identify potential differences in the dissolution profile of the pure drug and physical mixture of SD was also studied similarly, $900 \mathrm{~mL}$ of $0.1 \mathrm{~N} \mathrm{HCl}$ was used. Samples were collected at $0,2,4,6,8,10,15,30,40$, and 60 minutes using an automatic sampler type Distek Evolution 4300 and analyzed immediately after sampling, according to an appropriately validated UV method, at $266.5 \mathrm{~nm}$, using a UV-Vis spectrophotometer type Shimadzu 1700 (Kyoto, Japan). Each test was performed in triplicate and represented as mean and standard deviation ${ }^{5},{ }^{17}$.

\subsection{In-vivo pharmacokinetic studies}

For evaluating SDs effect on the drug's in-vivo bioavailability, relative bioavailability by comparing with the drug solution as a reference with $\mathrm{F} 1$ and $\mathrm{F} 2$ was studied in rats. The experiments were conducted after the approval from CPCSEA (Committee for prevention control and supervision of experimental animal's guidelines) Faculty of Pharmacy, VNS Group of Institutions, Neelbud Bhopal Protocol No. PH/IAEC/VNS/2K12/23. ${ }^{18}$ 


\subsection{HPLC method for estimation of famotidine}

Numerous methods for FMT determination in biological samples have been referred to, and reviews have been published. High-performance liquid chromatography (HPLC) with either ultraviolet or fluorescence detection is the most common analytical method to determine FMT concentrations in biological fluids during pharmacokinetics studies ${ }^{19}$.

\subsection{Preparation of mobile phase}

The mobile phase consisted of acetonitrile: water $(70: 30 \mathrm{v} / \mathrm{v})$, the flow was isocratic. All the components were passed through a $0.22 \mu \mathrm{m}$ Millipore filter assembly and degassed by sonication before use.

\subsubsection{Method development}

The HPLC method for analysis of FMT in the formulation and biological samples was developed using acetonitrile and water as the mobile phase in the ratio of $(70: 30 \mathrm{v} / \mathrm{v})$ at $266 \mathrm{~nm}$ with a photodiode array detector. The new method is partially validated for the estimation of FMT in biological matrix $^{20}$.

\subsubsection{Preparation of stock and standard solutions}

FMT solution was prepared by dissolving $10.0 \mathrm{mg}$ of the drug in $10.0 \mathrm{ml}$ methanol as the primary stock solution. The secondary stock solution was prepared by taking $1 \mathrm{ml}$ of primary stock and diluting it up to $10.0 \mathrm{ml}$ by methanol to form a $100 \mu \mathrm{g} / \mathrm{ml}$ sub-stock solution. From solution of $100 \mu \mathrm{g} / \mathrm{ml}$ dilutions of $0.5,1.5,2.5,3.5,4.5,5.5,6.5$ and $7.5 \mu \mathrm{g} / \mathrm{ml}$ were prepared.

\subsubsection{Sample preparation (protein precipitation method)}

For sample preparation, $1.0 \mathrm{ml}$ of blood from the rat was collected in a heparinized collection tube. The blood samples were immediately centrifuged at $9468 \mathrm{~g}$ for 15 mins at 5 으, and plasma was stored at -20 ․ C. After collecting plasma, the proteins present in the plasma were precipitated by taking $100 \mu \mathrm{l}$ blank plasma, $100 \mu \mathrm{l}$ of HPLC water, and $800 \mu \mathrm{l}$ acetonitrile in an Eppendorf tube. After that, the sample was shaken for 5 mins and then centrifuged at $9468 \mathrm{~g}$ for $12 \mathrm{mins}$. The supernatant was transferred to another Eppendorf tube. The samples were filtered and analyzed by HPLC as blank plasma.

\subsubsection{Standard curve preparation in plasma}

Standard dilutions were made from the stock solution in the range of $0.5-7.5 \mu \mathrm{g} / \mathrm{ml}$ comprising of $100 \mu \mathrm{l}$ of blank serum, and $100 \mu \mathrm{l}$ of standard dilution were mixed with $800 \mu \mathrm{l}$ of acetonitrile and centrifuged at $9468 \mathrm{~g}$ for 15 mins. The supernatant layer was separated, and then $20 \mu \mathrm{l}$ was injected into the HPLC system ${ }^{19}$.

\subsection{Experimental design for relative bioavailability study}

A single-dose, randomized study was conducted on 16 male Wistar rats(150- $200 \mathrm{gms})$. The rats were randomly divided into four groups of four rats each $(n=4)$. All the rats fasted for $12 \mathrm{hr}$ with ad libitum access to water. Group-I was a standard group without drug administration; group II was considered positive control and received pure drug suspension. The other two groups, group III and $\mathrm{IV}$, received the test formulation (F1 and F2). Formulation F3 was screened out as per in-vitro results as compared to $\mathrm{F} 1$ and $\mathrm{F} 2$. The pure drug was administered at $15 \mathrm{mg} / \mathrm{kg}$ per animal by making their suspension in $0.5 \% \mathrm{CMC}$ through oral feeding canula. The SDs were suspended in $0.5 \% \mathrm{CMC}$ and administered at a dose equivalent to $15 \mathrm{mg} / \mathrm{kg}$ per animal. Heparinized blood sample $(0.5 \mathrm{ml})$ was withdrawn from retroorbital puncture into Eppendorf tubes containing at time intervals of $0,1,2,4$, 6,8 , and 24 hours. The method used is diagrammatically presented in brief in fig 1 . The blood sample was immediately centrifuged at $9468 \mathrm{~g}$ for $15 \mathrm{~min}$ at $5 \circ \mathrm{C}$, and plasma was stored at -20 으 until HPLC analysis ${ }^{13}$. 

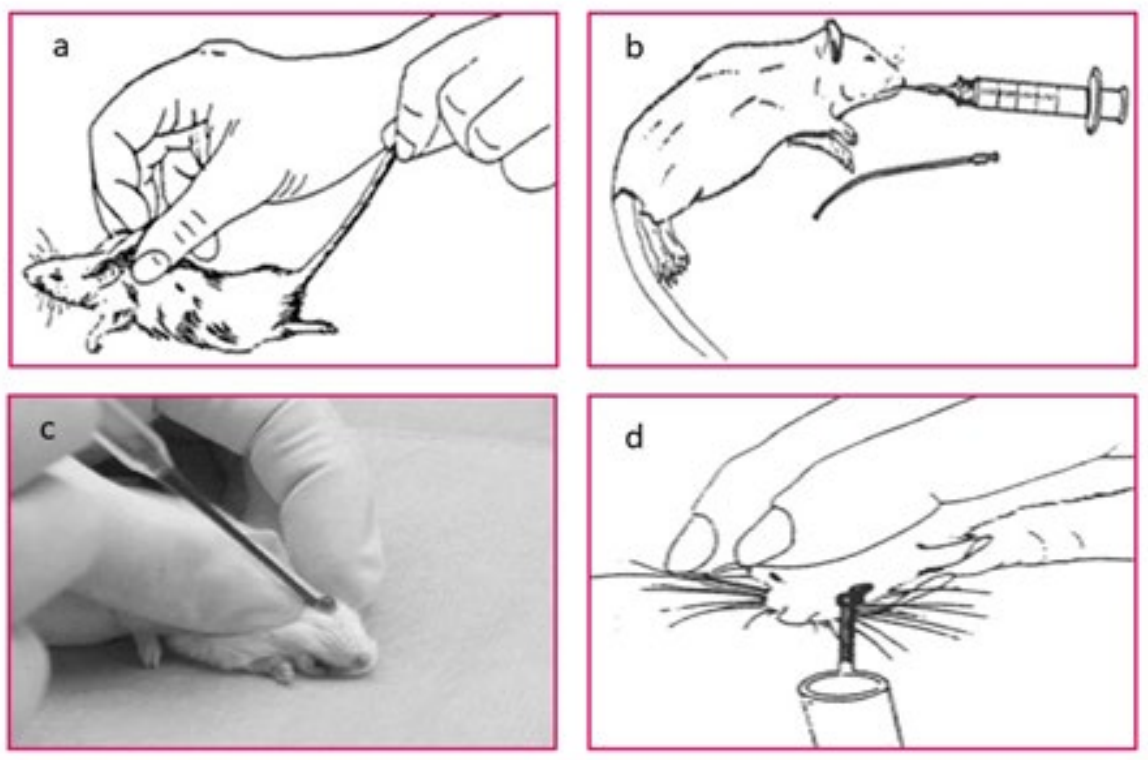

Fig 1. Diagrammatic representation of in-vivo bioavailability studies; a. Manual restraint of the rat; b. oral administration by rodent gavage needle; c. Retro-orbital puncture; d. blood collection

\subsection{Analysis of study samples}

After centrifugation samples collected at different time points were treated the same as blank plasma, i.e., $100 \mu \mathrm{l}$ plasma, $100 \mu \mathrm{l} \mathrm{HPLC}$ water, and $800 \mu \mathrm{l}$ of acetonitrile, then shaken for $5 \mathrm{~min}$, centrifuged. The supernatant was separated and analyzed by HPLC. The blank plasma sample showed no peaks at 6.03 mins, whereas plasma samples with the drug showed peaks at 6.03 mins.

\subsection{Statistical analysis:}

Statistical analysis was performed using Sigma Stat statistical software. Tukey's test was used to determine the difference between different formulations following ANOVA analysis. All results were considered statistically significant when $p<0.05$.

\section{Results and Discussions}

\subsection{Determination of percent drug content: Percent Drug content}

The drug content in F1, F2, and F3 SDs was found to be $91.59 \pm 1.47,82.72 \pm 2.41$, and $60.72 \pm 5.25 \%$, respectively, represented in Fig 2 . The data indicated that the drug content in the formulated solid dispersions (SD) was within the range of $60-90 \%$ of the theoretical amount, indicating the method used for the formulation was suitable and reproducible in nature ${ }^{21}$. Although the significantly less $(p<0.05)$ percent drug content in F3 may be related to less interaction between inulin and drug, it may be related to the high polar nature of inulin, leading to less interaction with less polar or nonpolar $\mathrm{FMT}^{22}$. 


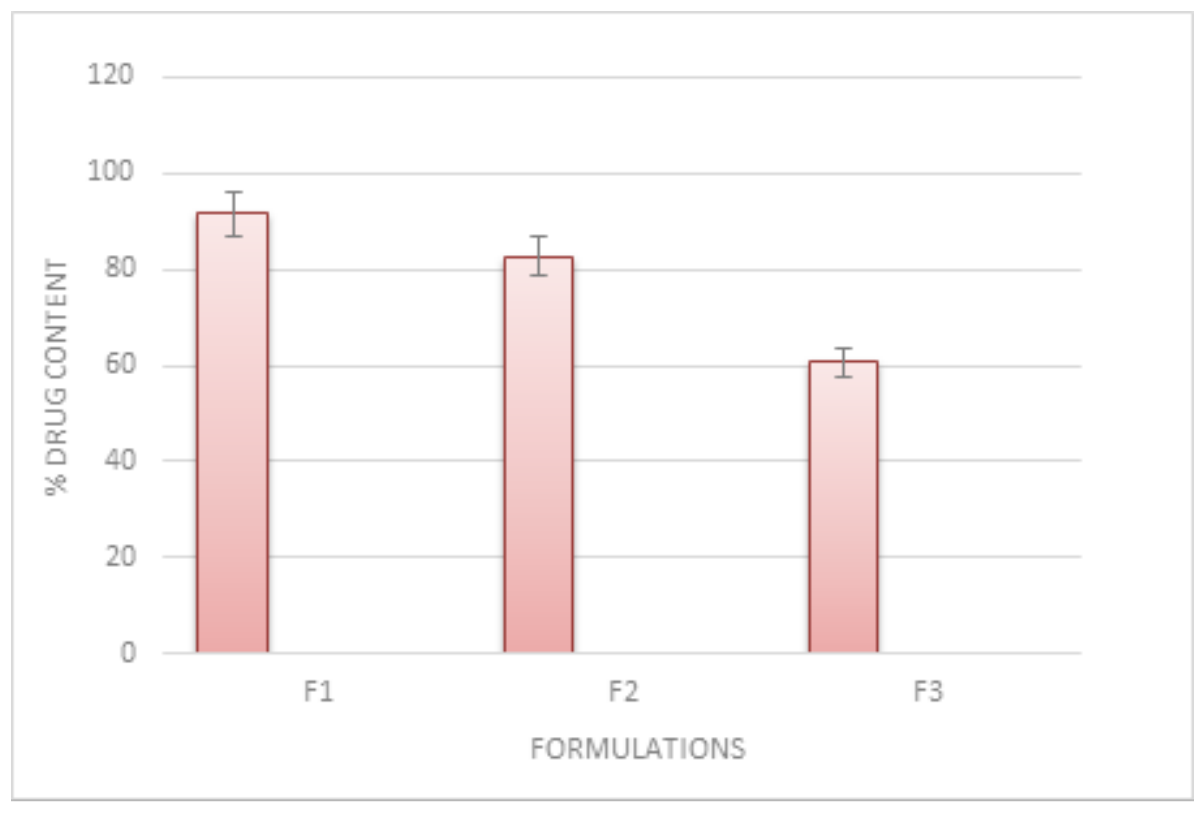

\section{Fig 2 - Percent drug content of SDs}

\subsection{Percent practical yield}

Percent practical yield of F1, F2, and F3 was $70.5 \pm 2.32,83.5 \pm 3.43$, and $74.5 \pm 2.56 \%$. SD prepared by milk powder showed a good percent practical yield because of its nonsticky nature after preparation ${ }^{23}$.

\subsection{Saturation solubility studies}

The solubility of formulations in distilled water, $0.1 \mathrm{~N} \mathrm{HCl}$, and ethanol were represented in table no. 1. The degree of supersaturation is defined as the concentration (C) of drug dissolved in the dissolution media divided by the equilibrium solubility (Ceq) of crystalline FMT in the dissolution media, that is, C/Ceq. The addition of polymers can increase FMT concentration in solution, as table no. 1 revealed. Polymers with supersaturation stabilizing effect provide increased levels of FMT in solution excessing the equilibrium solubility through either physical and/or chemical interactions with FMT molecules that inhibit precipitation. The above results may be explained as polymers may inhibit nucleation or crystal growth by adsorption on the drug crystalline interface, thereby preventing the crystal growth. PVP K-30 and milk showed an excellent concentration enhancing effect, which was in good agreement with other report ${ }^{24},{ }^{25}$, and our further observation involving drug release further confirmed our formulation optimization. 
Table No. 1 - Saturation solubility data of FMT, F1, F2, and F3

\begin{tabular}{|c|c|c|c|c|}
\hline Time (mins) & Famotidine & F1 & F2 & F3 \\
\hline \multicolumn{5}{|c|}{ Solubility in Water $(\mathrm{mg} / 5 \mathrm{ml})$} \\
\hline 10 & 0.11 & 2.34 & 1.99 & 0.401 \\
\hline 30 & 0.88 & 4.01 & 2.54 & 1.23 \\
\hline 60 & 1.23 & 8.34 & 7.65 & 5.98 \\
\hline 180 & 1.23 & 8.34 & 7.65 & 5.98 \\
\hline \multicolumn{5}{|c|}{ Solubility in $0.1 \mathrm{~N} \mathrm{HCl}(\mathrm{mg} / 5 \mathrm{ml})$} \\
\hline 10 & 2.21 & 2.63 & 1.84 & 0.59 \\
\hline 30 & 4.63 & 3.65 & 2.78 & 1.24 \\
\hline 60 & 5.12 & 8.78 & 6.87 & 6.56 \\
\hline 180 & 5.12 & 8.78 & 6.87 & 6.56 \\
\hline \multicolumn{5}{|c|}{ Solubility in ethanol $(\mathrm{mg} / 5 \mathrm{ml})$} \\
\hline 10 & 11.75 & 1.87 & 1.54 & 0.67 \\
\hline 30 & 12.11 & 4.65 & 3.56 & 1.78 \\
\hline 60 & 12.26 & 8.85 & 6.98 & 6.84 \\
\hline 180 & 12.26 & 8.85 & 6.99 & 6.84 \\
\hline
\end{tabular}

\subsection{FT-IR studies of FMT, physical mixture, and formulation:}

The FT-IR spectrum of pure FMT and that of the physical mixtures and optimum solid dispersions are shown in fig. 3. The spectrum of FMT showed characteristic bands at $3500 \mathrm{~cm}^{-1}$ (N-H stretching), $1637 \mathrm{~cm}^{-1}$ ( $\mathrm{C}=\mathrm{C}$ stretching), $1591 \mathrm{~cm}^{-1}$ (in-plane bending for $\left.\mathrm{N}-\mathrm{H}\right), 1278 \mathrm{~cm}^{-1}$ ( $\mathrm{C}-\mathrm{N}$ aromatic amine), $3020 \mathrm{~cm}^{-1}$ (C-H stretching), $671 \mathrm{~cm}^{-1}$ (=C-H bending) and $1154 \mathrm{~cm}^{-1}\left(\mathrm{~S}=\mathrm{O}\right.$ stretching) ${ }^{26}$. FTIR spectrum of the physical mixture of the drug and polymer showed no significant shift or reduction in the intensity of peaks of FMT at $1286 \mathrm{~cm}^{-1}$ for (C-N Stretching), $1531 \mathrm{~cm}^{-1}$ for ( $\mathrm{N}=\mathrm{H}$ bending), $3500 \mathrm{~cm}^{-1}$ (N-H stretching), and $1639 \mathrm{~cm}^{-1}$ (C=C stretching). FTIR spectrum of formulation showed some characteristic peaks of FMT such as $3423 \mathrm{~cm}^{-1}$ for (N-H stretching), $667 \mathrm{~cm}^{-1}$ (=C-H bending), 1670 $\mathrm{cm}^{-1}$ ( $\mathrm{C}=\mathrm{C}$ stretching), and $1286 \mathrm{~cm}^{-1}$ (C-N stretching). FTIR spectroscopic studies indicated that the drug is compatible with the polymer. 


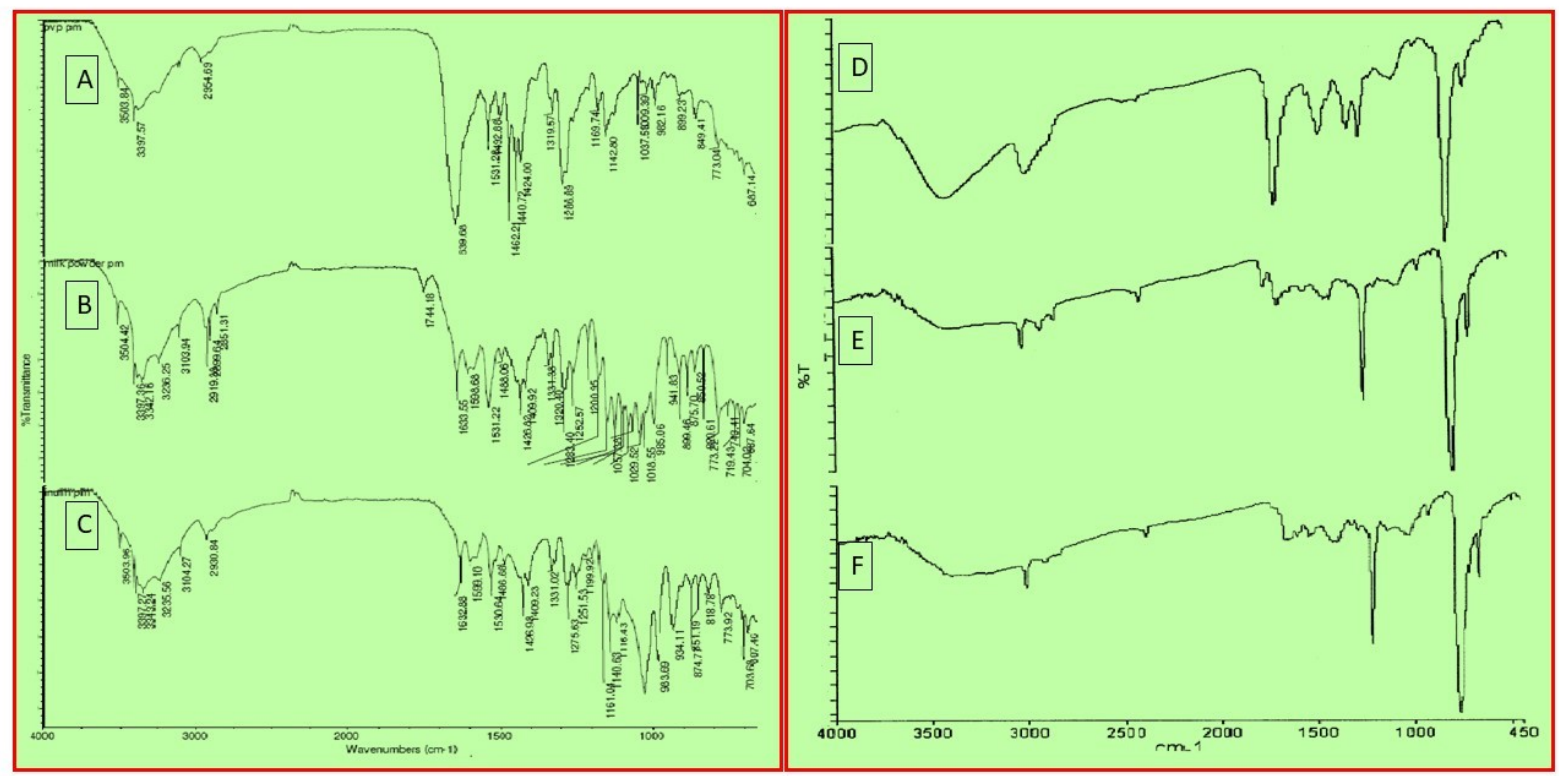

Fig 3: FT-IR spectrum of the physical mixture of A) FMT with PVP K-30; B) FMT with milk powder; C) FMT with inulin; D) F1; E) F2; F) F3.

\subsection{Powder X-ray diffraction (PXRD)}

The XRD pattern of FMT shows numerous sharp, narrow, and intense peaks at different angles, reflecting its high crystallinity. The spectrum of PVP-K30, milk powder, and inulin resemble a typical amorphous material devoid of any characteristic peaks. The solid dispersion formulations were shown to consist of broader peaks with lower intensity and a decrease in the number of peaks representing a decrease in crystallinity ${ }^{27}$. On comparison of the formulation pattern with that of pure drug, presented in Fig 4, it was observed that the number and intensity of peaks were found to be less in the formulation and decreased in the following order: Drug, F3, F2, and F1. 
Table 2 Fabric test methods

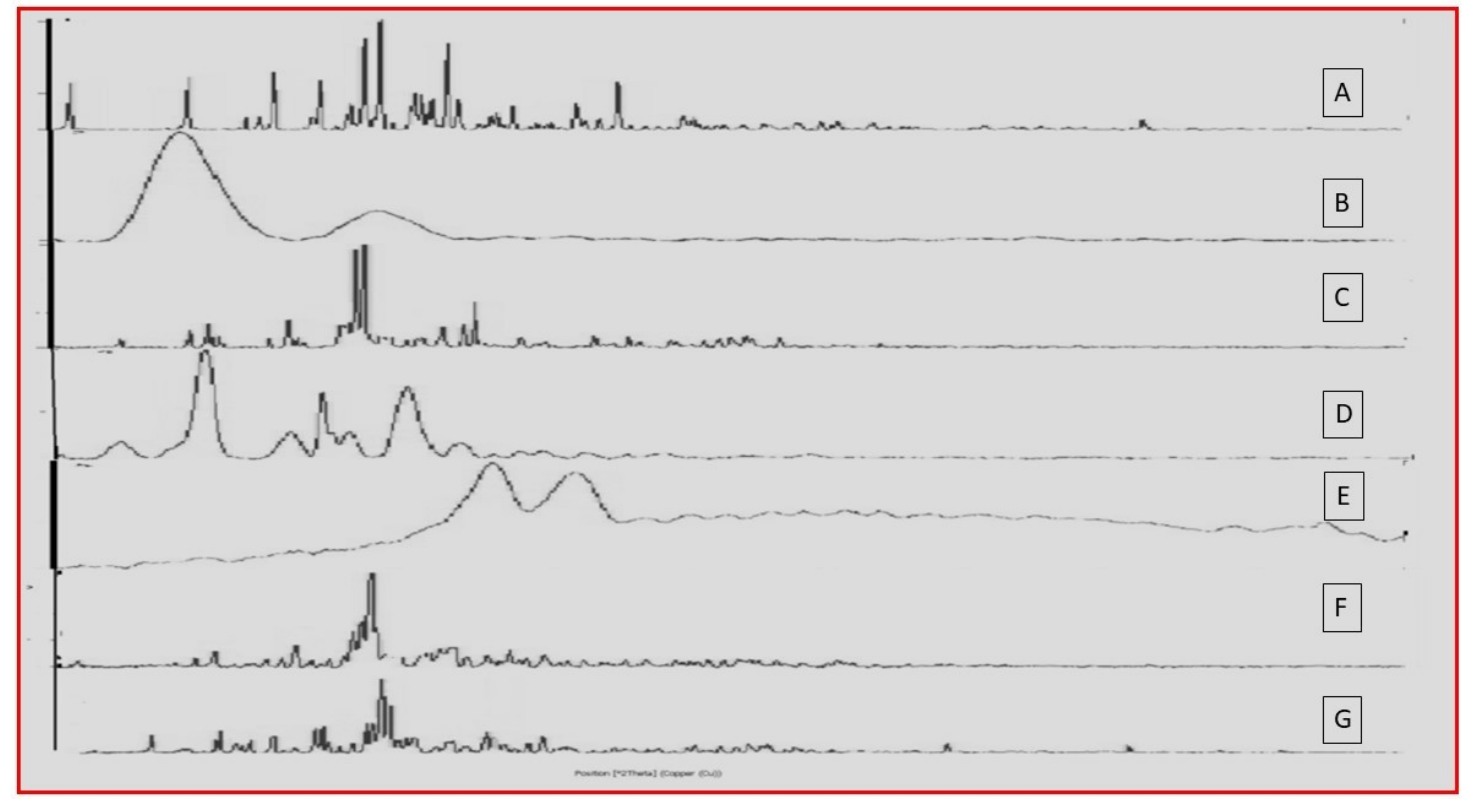

Fig 4: Powder -XRD of A) Drug; B) PVP-K30; C) Milk powder; D) Inulin; E) F-1; F) F-2; G) F-3.

\section{Fabric investigated}

The manufacturer's specification of the monofilament polyester fabric that was investigated is shown in Table 3 and, where applicable, the test method is included in parenthesis. The fabric investigated was a JG type (WF5505-JG) supplied by Asiatic Fiber Corporation and is widely utilised for garments in the pharmaceutical industry. However, without a detailed knowledge of fabrics and the test methods used, it is difficult to understand from the information given in Table 3 how well the fabric would perform in a pharmaceutical or healthcare cleanroom to the required parameters shown previously in Table 2.

\subsection{In-vitro dissolution study}

The results of in-vitro drug release studies in $0.1 \mathrm{~N} \mathrm{HCl}$ for $1 \mathrm{hr}$ are shown in fig 5 . In this study, the complete release of FMT observed with sustained supersaturation of drug over one hour was achieved using different amorphous polymers.

The rate of release from different formulation was initially higher, followed by slower release. The pure drug showed dissolution of $50.0 \%$ at the end of $1 \mathrm{hr}$, while SDs showed about $99.4 \%, 91.6 \%$, and $58.8 \%$ in PVP K 30 , milk powder, and inulin. The percent drug dissolution increased in SDs with PVP K-30 and milk powder as these polymers convert the crystalline nature of the drug to amorphous. These water-soluble carriers may operate in the microenvironment (diffusion layer) immediately surrounding the drug particles in the early stage of dissolution since the carrier completely dissolves in a short time, enhancing the solubility. Dissolution of FMT showed significant improvement by SDs (P-value $<0.05)$. By solid dispersion, the dissolution rate was 1.98 times higher than the pure drug at the end of the study. The reasons for the enhancement in drug dissolution could be the dispersion of the drug in pores of PVP K-30, milk powder, and inulin. The drug dissolution and solubility enhancement mechanism proposed in this study is 'spring and parachute,' 
where amorphous drugs are often rapidly released upon dissolution, and the subsequent drop of supersaturation is caused by drug crystallization ${ }^{28}$. With the outcome of this study, we can propose that such immediate release of the drug could be tuned by the nature of the polymer, which makes these solid dispersions promising candidate as a carrier for enhancing the solubility of FMT and other poorly water-soluble drugs.

Each point represents the mean \pm SD $(n=3)$

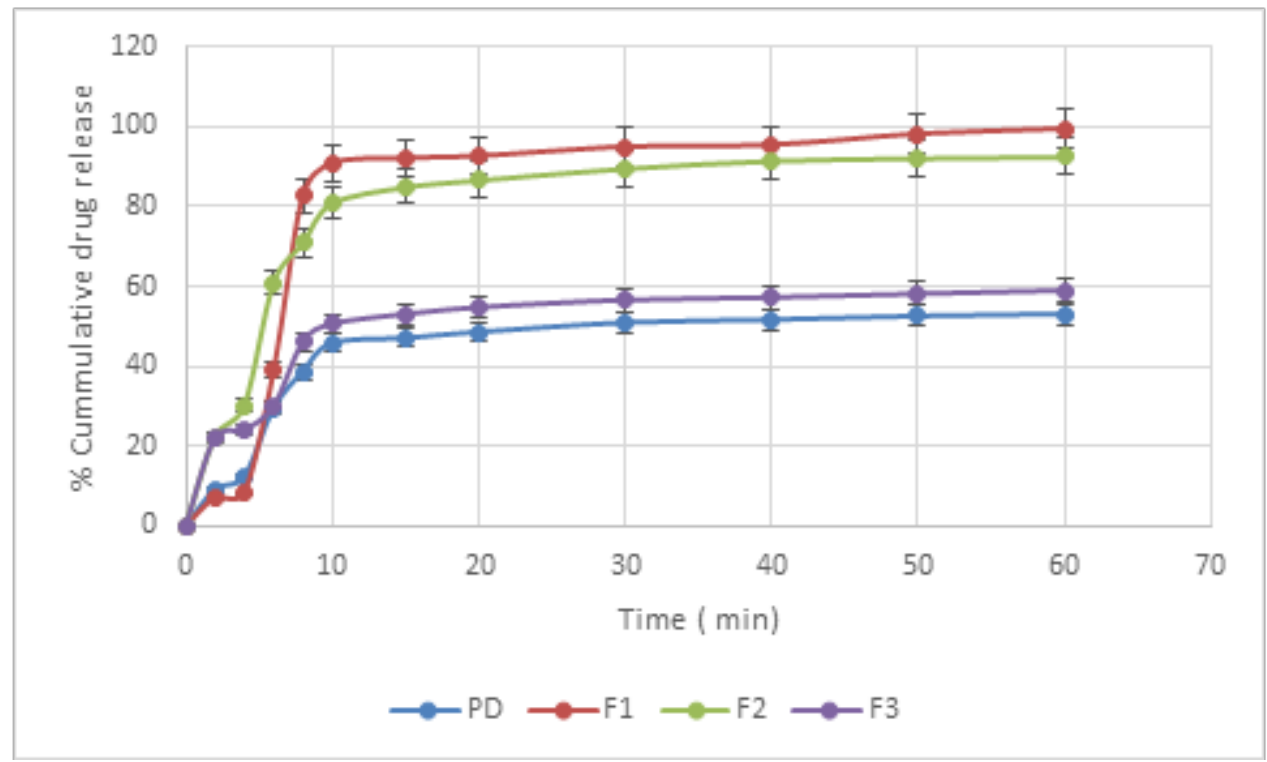

Fig 5: In-vitro drug release of famotidine, F1, F2, and F3

\subsection{In-vivo pharmacokinetic studies}

The plasma-drug concentration profiles of FMT after single-dose oral administration of each formulation drug were analyzed by reverse-phase HPLC method as described in materials and methods. Chromatograms are presented in fig. 6 , and the corresponding chromatographic data is summarized in Table No 2 . The standard calibration curve and regression coefficient are presented in fig. 7.

The various parameters of the plasma-drug concentration profile are presented in fig 8 and summarized in table no. 3. Plasma-drug concentration profiles of group III \& IV, i.e., F1 and F2 SDs, showed much higher drug concentration ( $\mathrm{Cmax}$ ) than group II, i.e., similarly administered FMT. These are the evidence of showing a high supersaturation degree of FMT in-vitro by SDs, which results in excellent absorption of the FMT in-vivo. Group III, i.e., showed the shortest Tmax ( $2 \mathrm{~h}$ ), which was in agreement with the in vitro dissolution study results and other works of literature, and was due to the rapid onset of supersaturation ${ }^{29}$. Since PVP K-30 is a pH stable polymer, which dissolves at $\mathrm{pH}>5$, the Tmax found in group III was short. The pharmacokinetic parameters, namely, maximum plasma concentration ( $\mathrm{Cmax}$ ) and time to reach $\mathrm{Cmax}$ (Tmax), were obtained directly from the data. The WinNonlin software trial version calculated the area under the plasma concentrationtime curve from 0 to the last measurable concentration (AUCO-24).

The significant enhancement in oral absorption of FMT for both F1 and F2 was due to the amorphous SD, which provided a rapid increase in free-drug concentration and the ability to maintain enhanced drug supersaturation levels relatively long time in the upper gastrointestinal 
tract. In the present study, significant enhancement of oral bioavailability was achieved with PVP K 30 (polymer), milk powder (food material), and inulin (polysaccharides) excipients through the solvent evaporation method.

Among the various carriers, PVP K-30 showed significantly higher bioavailability, both rate, and extent, than others, which suggests the use of PVP K-30 as a suitable synthetic polymer. Milk powder also showed enhancement of bioavailability and, therefore, can be suggested as a natural carrier.
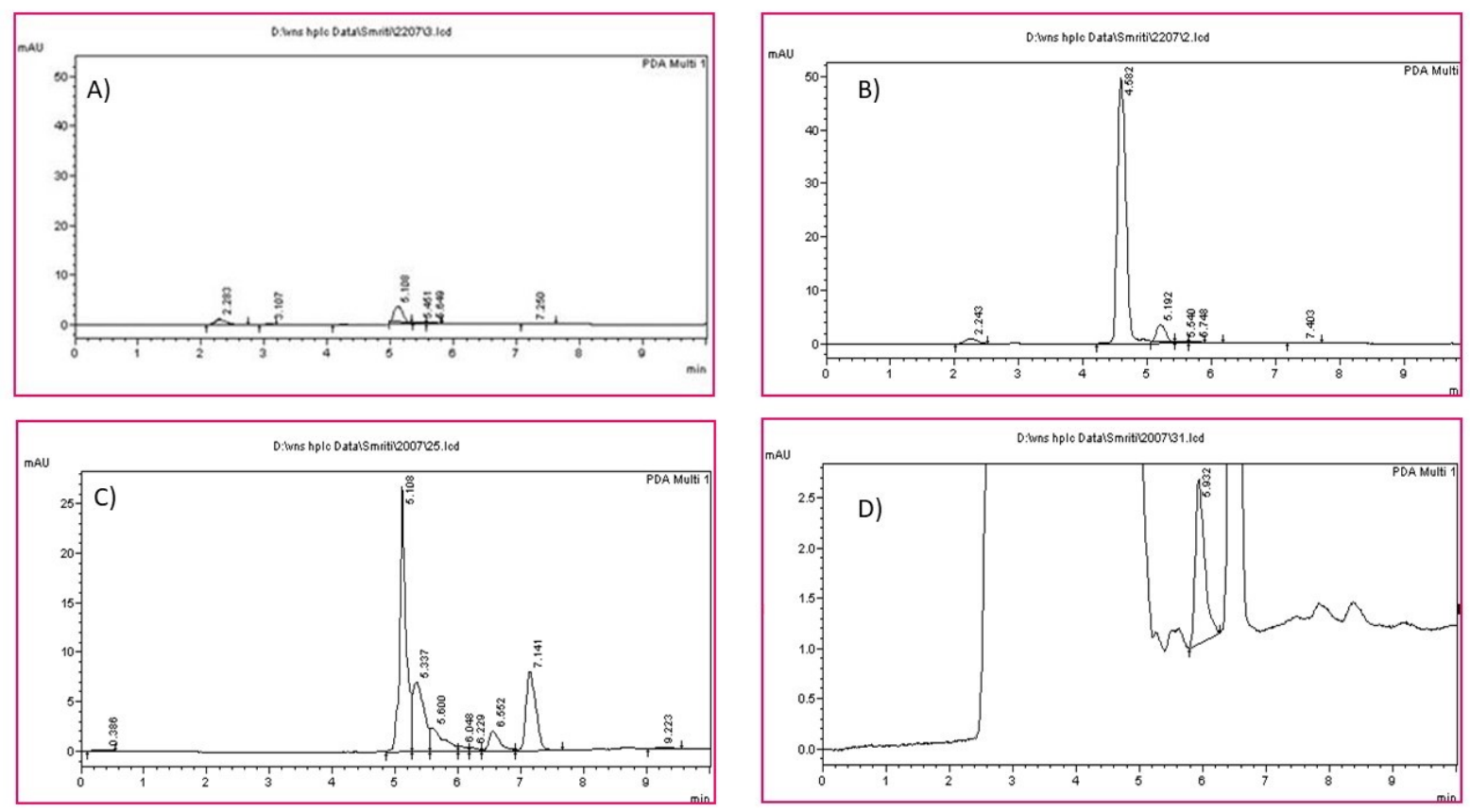

Fig 6: Chromatograms A) Methanol; B) Famotidine; C) Blank plasma; D) Drug in plasma

Table 2: Observation and calculation of chromatograms

\begin{tabular}{|l|l|l|l|l|l|l|l|}
\hline Sr. & $\begin{array}{l}\text { Conc. } \\
\text { No }\end{array}$ & Peak area & Peak area & Peak area & Mean & Stdev & $\begin{array}{l}\text { RSD } \\
\%\end{array}$ \\
\hline 1. & 0.5 & 217.1 & 223.2 & 230 & 223.43 & 6.453 & 2.89 \\
\hline 2. & 1.5 & 6541.3 & 6890.6 & 6734.4 & 6722.1 & 174.97 & 2.58 \\
\hline 3. & 2.5 & 10902.16 & 11234.6 & 11034.4 & 11057.05 & 167.37 & 1.51 \\
\hline 4. & 3.5 & 15263.6 & 15453.6 & 15197.7 & 15304.97 & 132.87 & 0.86 \\
\hline 5. & 4.5 & 19623.9 & 19891.4 & 19914.5 & 19809.93 & 161.52 & 0.81 \\
\hline 6. & 5.5 & 23984.7 & 23654.7 & 24100.5 & 23913.3 & 231.31 & 0.96 \\
\hline 7. & 6.5 & 28345.6 & 28598.6 & 28545.6 & 28496.6 & 133.42 & 0.46 \\
\hline 8. & 7.5 & 32706.5 & 32987.7 & 32643.5 & 32779.23 & 183.26 & 0.55 \\
\hline
\end{tabular}




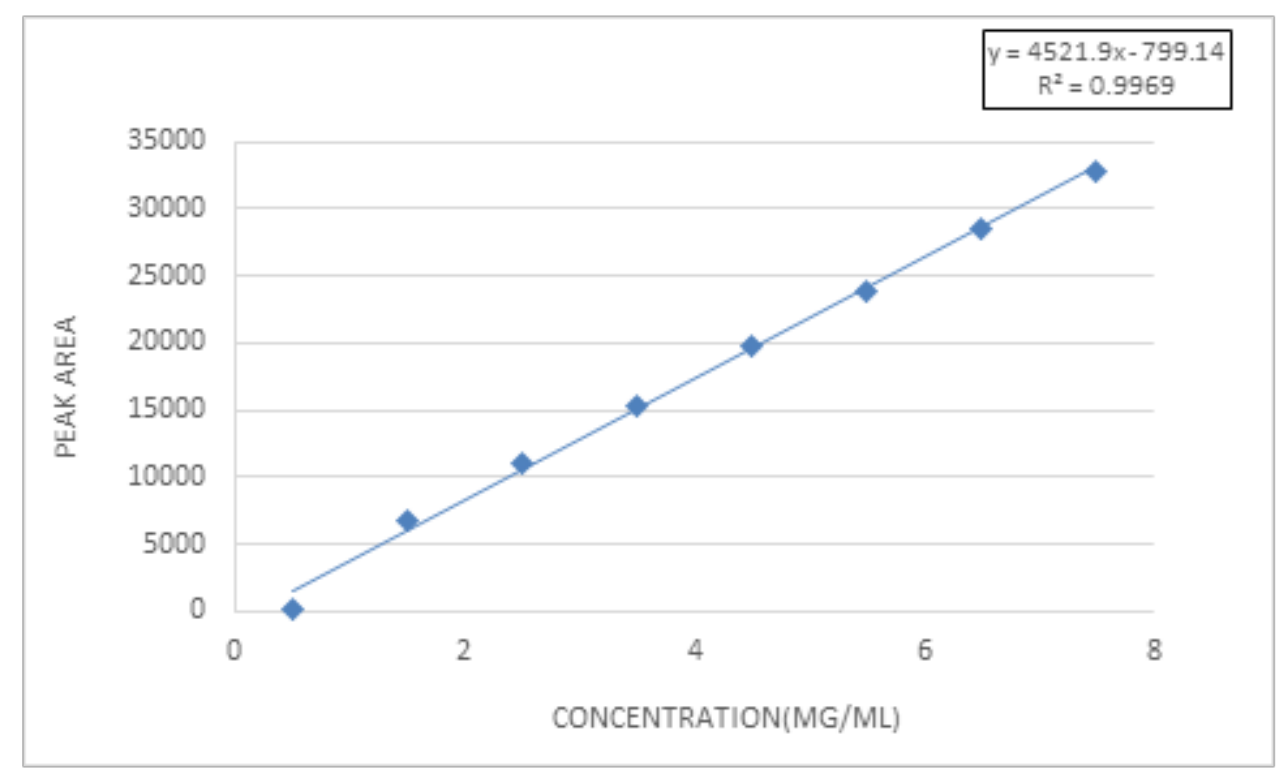

Fig 7: Standard curve of famotidine in plasma

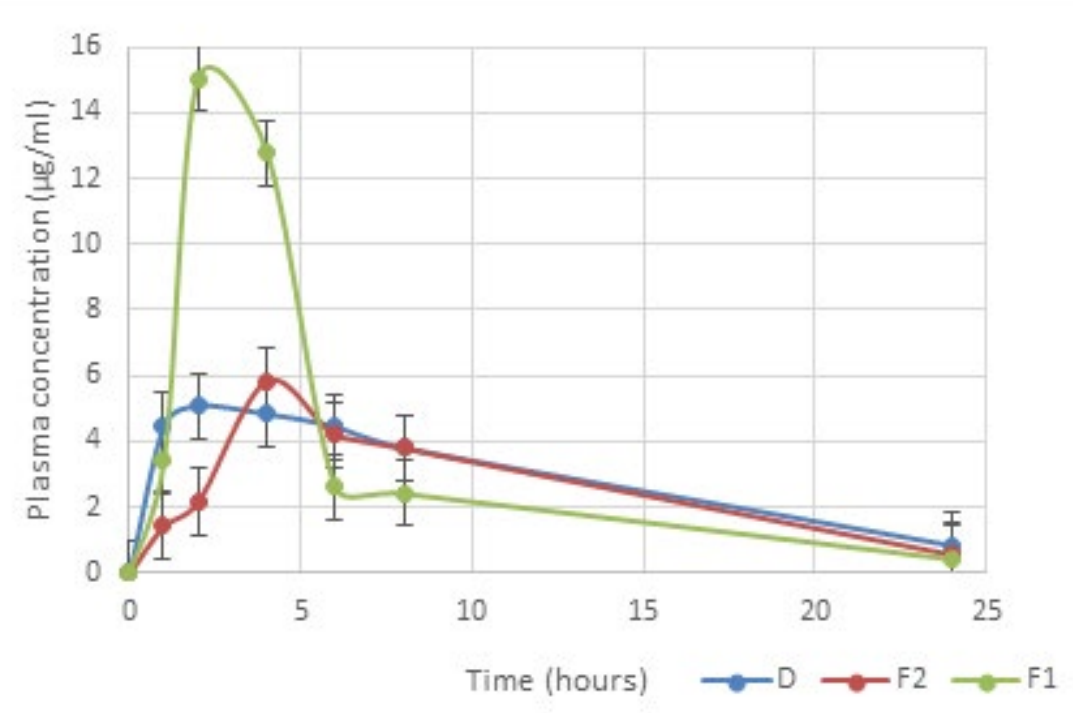

Figure 8: FMT plasma concentration vs. time profiles (mean \pm S.D) following oral administration of the pure drug and formulations 1 and $2(n=3)$.

Table 3: Pharmacokinetic parameters of FMT (mean \pm S.D.) following oral administration of the pure drug, formulations 1 and $2(n=3)$

\begin{tabular}{|l|l|l|l|}
\hline Parameters & Pure drug & $\mathrm{F}-1$ & $\mathrm{~F}-2$ \\
\hline$C_{\max }(\mu \mathrm{g} / \mathrm{ml})$ & $5.07 \pm 0.78$ & $15.05 \pm 2.45$ & $5.84 \pm 0.96$ \\
\hline $\mathrm{t}_{\max }(\mathrm{hr})$ & 2 & 2 & 4 \\
\hline $\mathrm{oAUC}^{24}(\mu \mathrm{g} . \mathrm{hr} / \mathrm{ml})$ & $16.46 \pm 2.78$ & $33.78 \pm 7.3$ & $21.52 \pm 4.5$ \\
\hline
\end{tabular}




\section{Conclusion}

The solubility and dissolution rate of FMT can be enhanced by using SDs of FMT with PVP-K 30 , milk powder, and inulin by a solvent evaporation technique. The solubilization effect of PVP-K 30 , reduction of particle aggregation of the drug, absence of crystallinity, and alteration of the surface properties of the drug particles are responsible for the enhanced solubility and dissolution rate of FMT from its SDs. From FTIR and PXRD spectroscopy, it was concluded that the molecular properties of drug and polymer suggested complexation between drug and polymer, demonstrated the formation of solid dispersion. It can be concluded that the preparation SDs of FMT with PVP-K 30, milk powder, and inulin provides a promising way to enhance its solubility and dissolution rate.

Conflict of Interest - Authors do not have any conflict of interest. 


\section{References}

1. Jain S, Jain S, Mishra A, Garg G, Modi RK. Formulation and characterization of fast disintegrating tablets containing Cefdinir solid dispersion. International Journal of Pharmacy \& Life Sciences 2012 Dec 1;3(12); 2190-2199.

2. Zhang $X$, Xing $H$, Zhao $Y, M a Z$. Pharmaceutical dispersion techniques for dissolution and bioavailability enhancement of poorly water-soluble drugs. Pharmaceutics 2018 Sep;10(3); 74.

3. Srinarong P, de Waard H, Frijlink HW, Hinrichs WL. Improved dissolution behavior of lipophilic drugs by solid dispersions: the production process as starting point for formulation considerations. Expert opinion on drug delivery 2011 Sep 1;8(9):1121-1140.

4. Van Duong T, Van den Mooter G. The role of the carrier in the formulation of pharmaceutical solid dispersions. Part II: amorphous carriers. Expert opinion on drug delivery 2016 Dec 1; 13(12): 1681-1694.

5. Milne $M$, Liebenberg $W$, Aucamp $M$. The stabilization of amorphous zopiclone in an amorphous solid dispersion. Aaps Pharmscitech 2015 Oct 1;16(5):1190-1202.

6. Joe JH, Lee WM, Park YJ, Joe KH, Oh DH, Seo YG, Woo JS, Yong CS, Choi HG. Effect of the soliddispersion method on the solubility and crystalline property of tacrolimus. International journal of pharmaceutics 2010 Aug 16; 395(1-2):161-166.

7. Huang $Y$, Zhao $X, Z u Y$, Wang L, Deng $Y$, Wu M, Wang $\mathrm{H}$. Enhanced solubility and bioavailability of apigenin via preparation of solid dispersions of mesoporous silica nanoparticles. Iranian journal of pharmaceutical research: IJPR 2019; 18(1): 168.

8. Van Duong T, Van den Mooter G. The role of the carrier in the formulation of pharmaceutical solid dispersions. Part II: amorphous carriers. Expert opinion on drug delivery 2016 Dec 1; 13(12): 1681-1694.

9. Dong $W$, Su X, Xu M, Hu M, Sun Y, Zhang P. Preparation, characterization, and in vitro/vivo evaluation of polymer-assisting formulation of atorvastatin calcium based on solid dispersion technique. Asian journal of pharmaceutical sciences 2018 Nov 1; 13(6): 546-554.

10. Tekade AR, Yadav JN. A Review on Solid Dispersion and Carriers Used Therein forSolubility Enhancement of Poorly Water Soluble Drugs. Advanced Pharmaceutical Bulletin 2020 May 11; 10(3): 359-369.

11. Teoh XY, bt Mahyuddin FN, Ahmad W, Chan SY. Formulation strategy of nitrofurantoin: cocrystal or solid dispersion?. Pharmaceutical Development and Technology 2020 Feb 7; 25(2): 245-251.

12. Zhang D, Lin J, Zhang F, Han X, Han L, Yang M, Zou W. Preparation and evaluation of andrographolide solid dispersion vectored by silicon dioxide. Pharmacognosy Magazine 2016 May; 12(Suppl 2): S245.

13. Simonazzi A, Cid AG, Paredes AJ, Schofs L, Gonzo EE, Palma SD, Bermúdez JM. Development and in vitro evaluation of solid dispersions as strategy to improve albendazole biopharmaceutical behavior. Therapeutic delivery 2018 Sep; 9(9): 623-638. 
14. Castro SG, Bruni SS, Lanusse CE, Allemandi DA, Palma SD. Improved albendazole dissolution rate in pluronic 188 solid dispersions. Aaps Pharmscitech 2010 Dec 1; 11(4): 1518-1525.

15. Paul G, Bisio C, Braschi I, Cossi M, Gatti G, Gianotti E, Marchese L. Combined solid-state NMR, FTIR and computational studies on layered and porous materials. Chemical Society Reviews 2018; 47(15): 5684-5739.

16. Huang BB, Liu DX, Liu DK, Wu G. Application of Solid Dispersion Technique to Improve Solubility and Sustain Release of Emamectin Benzoate. Molecules 2019 Jan; 24(23): 4315.

17. Papageorgiou GZ, Bikiaris D, Karavas E, Politis S, Docoslis A, Park Y, Stergiou A, Georgarakis E. Effect of physical state and particle size distribution on dissolution enhancement of nimodipine/PEG solid dispersions prepared by melt mixing and solvent evaporation. The AAPS journal 2006 Dec 1; 8(4): E623-631.

18. Shuai S, Yue S, Huang Q, Wang W, Yang J, Lan K, Ye L. Preparation, characterization and in vitro/vivo evaluation of tectorigenin solid dispersion with improved dissolution and bioavailability. European journal of drug metabolism and pharmacokinetics 2016 Aug 1 ; 41(4): 413-422.

19. Dewani AP, Tripathi AS, Shelke PG, Bakal RL, Mohale DS, Chandewar AV. Development of solidphase extraction and HPLC method for simultaneous estimation of ilaprazole and glimepiride in rat plasma: Application to pharmacokinetic studies. Journal of chromatographic science 2017 Mar 1; 55(3): 327-333.

20. Li, J., et al., Determination of metrafenone in vegetables by matrix solid-phase dispersion and HPLC-UV method. Food Chem 2017. 214: 77-81.

21. Chaulang G, Patil K, Ghodke D, Khan S, Yeole P. Preparation and characterization of solid dispersion tablet of furosemide with crospovidone. Research journal of pharmacy and technology. 2008; 1(4): 386-389.

22. Visser MR, Baert L, van't Klooster G, Schueller L, Geldof M, Vanwelkenhuysen I, De Kock H, De Meyer S, Frijlink HW, Rosier J, Hinrichs WL. Inulin solid dispersion technology to improve the absorption of the BCS Class IV drug TMC240. European journal of pharmaceutics and biopharmaceutics 2010 Feb 1; 74(2): 233-8.

23. Fan W, Zhang X, Zhu W, Di L. The Preparation of Curcumin Sustained-Release Solid Dispersion by Hot-Melt Extrusion - II. Optimization of Preparation Process and Evaluation In Vitro and In Vivo. Journal of Pharmaceutical Sciences. 2020 Mar 1;109(3):1253-60.

24. Shi X, Huang W, Xu T, Fan B, Sheng X. Investigation of Drug-Polymer Miscibility and Solubilization on Meloxicam Binary Solid Dispersion. Journal of Pharmaceutical Innovation. 2020 Mar;15(1):125-37.

25. Basha M, Salama A, Noshi SH. Soluplus ${ }^{\circledR}$ based solid dispersion as fast disintegrating tablets: a combined experimental approach for enhancing the dissolution and antiulcer efficacy of famotidine. Drug Development and Industrial Pharmacy 2020 Feb 1; 46(2): 253-263.

26. Jamrógiewicz $M$, Milewska K. Studies on the influence of $\beta$-cyclodextrin derivatives on the physical stability of famotidine. Spectrochimica Acta Part A: Molecular and Biomolecular Spectroscopy 2019 Aug 5; 219: 346-357. 
27. Ding Z, Wang L, Xing Y, Zhao Y, Wang Z, Han J. Enhanced oral bioavailability of celecoxib nanocrystalline solid dispersion based on wet media milling technique: formulation, optimization and in vitro/in vivo evaluation Pharmaceutics 2019 Jul; 11(7): 328-330.

28. Shi NQ, Wang SR, Zhang Y, Huo JS, Wang LN, Cai JH, Li ZQ, Xiang B, Qi XR. Hot melt extrusion technology for improved dissolution, solubility and "spring-parachute" processes of amorphous self-micellizing solid dispersions containing BCS II drugs indomethacin and fenofibrate: Profiles and mechanisms. European Journal of Pharmaceutical Sciences 2019 Mar 15; 130: 78-90.

29. El-Shaheny R, Radwan MO, Belal F, Yamada K. Pentabromobenzyl-RP versus triazole-HILIC columns for separation of the polar basic analytes famotidine and famotidone: LC method development combined with in silico tools to follow the potential consequences of famotidine gastric instability. Journal of Pharmaceutical and Biomedical Analysis 2020 Apr 18: 113305. 